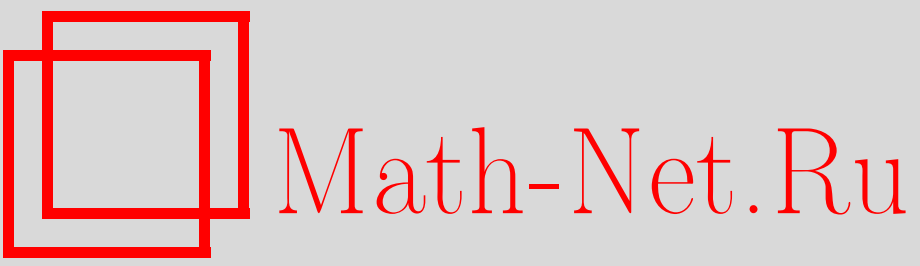

В. А. Петров, О фазе парциальных амплитуд вне массовой поверхности, ТМФ, 2001, том 129, номер 1, 103-105

DOI: https://doi.org/10.4213/tmf522

Использование Общероссийского математического портала Math-Net.Ru подразумевает, что вы прочитали и согласны с пользовательским соглашением

http://www.mathnet.ru/rus/agreement

Параметры загрузки:

IP : 54.237 .206 .68

26 апреля 2023 г., 11:39:14 


\title{
ТЕОРЕТИЧЕСКАЯ
}

И МАТЕМАТИЧЕСКАЯ

ФИЗИКА

Том 129, № 1

октябрь, 2001

(C) 2001 г.

\author{
В.А. Петров*
}

\section{О ФАЗЕ ПАРЦИАЛЬНЫХ АМПЛИТУД ВНЕ МАССОВОЙ ПОВЕРХНОСТИ}

Показано, что фаза парциальной амплитуды в области упругого рассеяния с одной частицей вне массовой поверхности не зависит от ее виртуальности.

1. Рассмотрим запаздываюшую амплитуду рассеяния двух бесспиновых частиц $a$ и $b$ с массами $m$ и $M$

$$
T_{a b}^{\mathrm{R}}\left(q^{\prime}, p^{\prime} \mid q, p\right)=-\int d x e^{i Q x}\left\langle p^{\prime}\left|\frac{\delta J_{a}\left(-\frac{x}{2}\right)}{\delta \phi_{a}^{+}\left(\frac{x}{2}\right)}\right| p\right\rangle,
$$

где $\phi_{a}$ - поле, отвечающее частицам $a, J_{a}(x)=i\left(\delta S / \delta \phi_{a}(x)\right) S^{+}, Q=\left(q^{\prime}+q\right) / 2$.

Из обобшенной (вне массовой поверхности) унитарности $S$-матрицы [1] следует, что

$$
\frac{\delta J_{a}(x)}{\delta \phi_{a}^{+}(y)}-\frac{\delta J_{a}^{+}(y)}{\delta \phi_{a}(x)}=i\left[J_{a}(x), J_{a}^{+}(y)\right]
$$

или для матричных элементов, отвечаюших нашему случаю $\left(T^{\mathrm{A}}-\right.$ опережаюшая амплитуда),

$$
T_{a b}^{\mathrm{R}}\left(q^{\prime}, p^{\prime} \mid q, p\right)-T_{a b}^{\mathrm{A}}\left(q^{\prime}, p^{\prime} \mid q, p\right)=i \int d x e^{i Q x}\left\langle p^{\prime} \mid\left[J_{a}\left(-\frac{x}{2}\right), J_{a}^{+}\left(\frac{x}{2}\right)\right] p\right\rangle .
$$

В дальнейшем рассматривается случай, когда частицы $a$ находятся вне массовой поверхности,

$$
\begin{aligned}
& -V<q^{\prime 2} \equiv \zeta^{\prime}<m^{2}, \\
& -V<q^{\prime 2} \equiv \zeta<m^{2},
\end{aligned}
$$

где $V$ - фиксированное, но произвольное положительное число. В книге [1] было доказано, что в этом случае гарантируется возможность использования интегральных фурье-представлений.

\footnotetext{
* Институт физики высоких энергий, г. Протвино, Московская обл., Россия
} 
2. Нетрудно убедиться в том, что

$$
T_{a b}^{\mathrm{A}}\left(q_{0}^{\prime}, \vec{q}^{\prime} ; \vec{p}^{\prime} \mid q_{0}, \vec{q} ; \vec{p}\right)=\overline{T_{a b}^{\mathrm{R}}}\left(q_{0}^{\prime},-\vec{q}^{\prime} ;-\vec{p}^{\prime} \mid q_{0},-\vec{q} ;-\vec{p}\right)
$$

Действительно,

$$
\begin{gathered}
\overline{T_{a b}^{\mathrm{R}}\left(\tilde{q}^{\prime}, \tilde{p}^{\prime} \mid \tilde{q}, \tilde{p}\right)}=- \\
\tilde{a} \equiv\left(a_{0},-\vec{a}\right) .
\end{gathered}
$$

Далее, пусть $T$ - антиунитарный оператор обрашения времени. Тогда по определению имеем

$$
\begin{aligned}
\left\langle\tilde{p}\left|\frac{\delta J_{a}^{+}\left(\frac{x}{2}\right)}{\delta \phi_{a}\left(-\frac{x}{2}\right)}\right| \tilde{p}^{\prime}\right\rangle & =\left\langle p^{\prime}\left|T \frac{\delta J_{a}\left(\frac{x}{2}\right)}{\delta \phi_{a}^{+}\left(-\frac{x}{2}\right)} T^{-1}\right| p\right\rangle= \\
& =\left\langle p^{\prime}\left|\frac{\delta J_{a}^{+}\left(\frac{\tilde{x}}{2}\right)}{\delta \phi_{a}\left(-\frac{\tilde{x}}{2}\right)}\right| p\right\rangle
\end{aligned}
$$

откуда и следует соотношение (3).

С учетом лоренц-инвариантности получим из формулы (1) $\left(T_{a b}^{\mathrm{R}} \equiv T_{a b}\right)$

$$
\begin{aligned}
& T_{a b}\left(s, \vec{n}^{\prime} \cdot \vec{n} ; \zeta^{\prime}, \zeta\right)-\overline{T_{a b}}\left(s, \vec{n}^{\prime} \cdot \vec{n} ; \zeta^{\prime}, \zeta\right)= \\
& \quad=i \frac{\lambda^{\frac{1}{2}}\left(s, m^{2}, M^{2}\right)}{32 s \pi^{2}} \int d \vec{e} \overline{T_{a b}}\left(s, \vec{e} \cdot \vec{n}^{\prime} ; \zeta^{\prime}, m^{2}\right) T_{a b}\left(s, \vec{n} \cdot \vec{e} ; \zeta, m^{2}\right),
\end{aligned}
$$

где в системе центра масс $\left(\vec{q}+\vec{p}=\vec{q}^{\prime}+\vec{p}^{\prime}=0\right) \vec{n}^{\prime}=\vec{p} /\left|\vec{p}^{\prime}\right|, \vec{n}=\vec{p} /|\vec{p}|, \vec{e}$ - единичный вектор промежуточного физического (т.е. на массовой поверхности) состояния $(a, b)$, $\lambda(x, y, z)=x^{2}+y^{2}+z^{2}-2 x y-2 y z-2 x z$, а значение $s=(q+p)^{2}=\left(q^{\prime}+p^{\prime}\right)^{2}$ взято ниже первого неупругого порога. Мы предполагаем, что все неупругие пороги лежат выше, чем $(m+M)^{2}$.

Переходя к парциальным амплитудам

$$
T_{a b}\left(s, \vec{n}^{\prime} \cdot \vec{n} ; \zeta^{\prime}, \zeta\right)=\frac{18 \pi \sqrt{s}}{\lambda^{\frac{1}{2}}\left(s, m^{2}, M^{2}\right)} \sum_{l}(2 l+1) f_{l}\left(s ; \zeta^{\prime}, \zeta\right) P_{l}\left(\vec{n}^{\prime} \vec{n}\right)
$$

где $P_{l}$ - полиномы Лежандра, имеем из формулы (1)

$$
\operatorname{Im} f_{l}\left(s ; \zeta^{\prime}, \zeta\right)=\overline{f_{l}}\left(s ; \zeta^{\prime}, m^{2}\right) f_{l}\left(s ; \zeta, m^{2}\right)
$$


3. Из последнего соотношения следует, что

$$
\frac{\operatorname{Re} f_{l}\left(s ; \zeta^{\prime}, m^{2}\right)}{\operatorname{Im} f_{l}\left(s ; \zeta^{\prime}, m^{2}\right)}=\frac{\operatorname{Re} f_{l}\left(s ; \zeta, m^{2}\right)}{\operatorname{Im} f_{l}\left(s ; \zeta, m^{2}\right)}
$$

т.е. фаза парциальной амплитуды $f_{l}\left(s ; \zeta, m^{2}\right)$ не зависит от величины $\zeta$, по крайней мере из интервала (2). Использованные выше соображения не дают возможности сделать такое же заключение относительно фазы амплитуды $f_{l}\left(s ; \zeta^{\prime}, \zeta\right)$ при $\zeta \neq m^{2}$.

Итак, мы показали, что при сделанных предположениях фаза парциальной амплитуды $f_{l}\left(s ; \zeta, m^{2}\right)$ при энергиях $\sqrt{s}$ ниже первого неупругого порога не зависит от виртуальности $\zeta$.

Благодарности. Автор благодарен за обсуждения Т. Т. Ву, А. А. Логунову и А. Мартену.

\section{Список литературы}

[1] Н. Н. Боголюбов, Б. В. Медведев, М. К. Поливанов. Вопросы теории дисперсионных соотношений. М.: Физматгиз, 1958.

Поступила в редакцию 4.XII.2000 г., после доработки 12.III.2001 г. 